\title{
EL CONSUMO ADOLESCENTE DE LA FICCIÓN SERIADA
}

\section{TEEN CONSUMPTION SERIOUS FICTION}

\section{AUTOR/ES}

Maddalena Fedele: Investigadora, GRISS (Grupo de Investigación en Imagen, Sonido y Síntesis). Departamento de Comunicación Audiovisual y Publicidad I. UAB (Universitat Autònoma de Barcelona) Catalunya (España) maddalena.fedele@uab.cat ; maddalena.fedele@gmail.com

Núria García-Muñoz: Investigadora, GRISS (Grupo de Investigación en Imagen, Sonido y Síntesis). Departamento de Comunicación Audiovisual y Publicidad I. UAB (Universitat Autònoma de Barcelona) Catalunya (España) nuria.garcia@uab.cat

\section{RESUMEN}

Se presentan los resultados de una investigación sobre el consumo adolescente de series y seriales televisivos dirigidos a una audiencia juvenil. La preferencia que los jóvenes otorgan a la ficción en su dieta televisiva ha animado la investigación sobre su consumo de ese macrogénero, sobre todo a partir de los años ochenta, coincidiendo con un aumento en la producción de ficción dirigida expresamente a los adolescentes. Hoy en día, para poder investigar el consumo juvenil de ficción, hay que tener en cuenta tanto el actual panorama multimediático, caracterizado por la convergencia y la digitalización, como el consumo multimediático y multitasking de los jóvenes.

El objetivo principal del estudio ha sido el de realizar una aproximación teórica al binomio adolescentes y teen series, consideradas como productos de ficción seriada dirigidos a un target juvenil. La aproximación a los estudios sobre recepción juvenil y ficción televisiva llevada a cabo se centra en las aportaciones realizadas en el periodo 1980-2009, en un contexto internacional. Se propone una categorización de los trabajos analizados, a partir de cinco grandes categorías: efecto cultivo, estudio de las motivaciones, apropiación de significados por parte de los jóvenes, construcción de las identidades y consumo y usos de las series.

\section{PALABRAS CLAVE}

Adolescentes - Jóvenes - Recepción - Consumo - Ficción

\section{ABSTRACT}

This article presents the results of a research about the adolescents' consumption of fiction series and serials targeted to a young audience. Young people's preference for fiction in television programming has stimulated researches about their consumption of this television macro genre, especially since the 1980s. It has also to be remarked the 
parallel increase in the production of television fiction targeted to teenagers. Nowadays, in order to investigate youth's consumption of television fiction, they have to be considered both the actual and complex multimedia environment, characterized by convergence and digitalization, and the multimedia and multitasking media consumption that young people are used to.

The principal aim of the research has been the realization of a theoretical approach about adolescents and teen series, considered as serial fiction products targeted to a young audience. The approach at the studies about the young people's television reception of the television fiction is based on the contributions realized in the period 1980-2009 in an international context. It is suggested a categorization of the studies according to five main categories: the cultivation effect, the study of motivations, the meanings appropriation by the young people, the construction of adolescents' identities, and the series consumption and use.

\section{KEY WORDS}

Adolescents - Young people - Reception - Consumption - Fiction

ÍNDICE

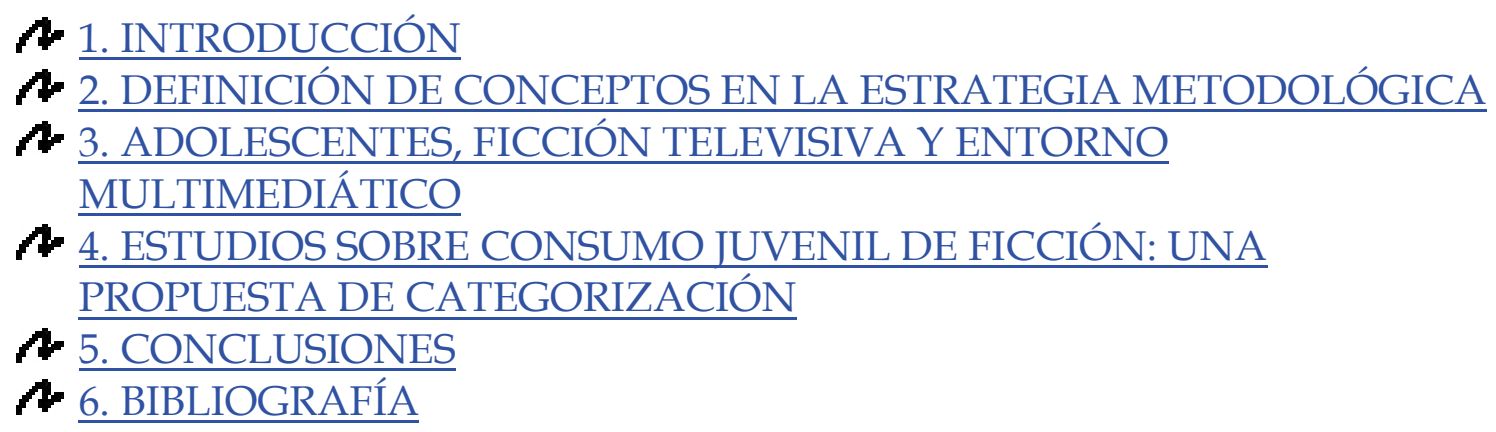

\section{INTRODUCCIÓN}

La relación entre los jóvenes y la televisión ha sido abordada por diversas disciplinas antes de la era digital y también en este nuevo escenario mediático, momento en el que aparecen nuevas formas de consumo que conviven con otras ya tradicionales. El conocimiento sobre los jóvenes y la ficción televisiva representa un área de interés que requiere cada vez una mayor atención por parte del mundo académico (Von Feilitzen, 2004). Este artículo presenta los resultados de un estudio centrado en el consumo adolescente de la ficción seriada televisiva. 
El objetivo principal del estudio ${ }^{1}$ ha sido realizar una aproximación teórica a la relación entre adolescentes y «teen series», definidas como productos de ficción seriada dirigida al «target» juvenil. La intención por tanto es la de establecer las características fundamentales de los estudios internacionales sobre el consumo juvenil de los productos de ficción seriada dirigidos a los jóvenes. Esta investigación se sitúa dentro de un marco teórico propio de los «audience studies», que enfatiza el concepto de audiencia activa y la importancia de los usos, tanto individuales como sociales, que las audiencias hacen de los medios. La recepción multimediática y "multitasking» de las audiencias juveniles, acostumbradas a vivir envueltas por una miríada de pantallas que utilizan constantemente en su vida cotidiana (Prado et al., 2006), forma parte del contexto del estudio. Estos jóvenes están acostumbrados a consumir cada día productos mediáticos producidos a menudo en ámbitos diferentes y lejanos de los contextos locales de recepción y consumo, $\mathrm{y}$, al mismo tiempo, están conectados constantemente al mundo, a través de Internet, de los móviles, de las cadenas televisivas globales, etc. Se trate o no de la aldea global pronosticada por Mcluhan, el ambiente vital en el que estas generaciones están sumergidas tiene valías globales y globalizadoras (Suess et al., 1998), pese a que en determinados contextos y antes productos específicos se observa un interés de esta audiencia hacia historias desarrolladas en ámbitos más locales y cercanos (Lemish et al., 1998).

\section{DEFINICIÓN DE CONCEPTOS EN LA ESTRATEGIA METODOLÓGICA}

Las «teen series» se han analizado en relación al más amplio conjunto de la ficción, entendida como un macrogénero de la programación televisiva a partir de la metodología de Euromonitor ${ }^{2}$. Los antecedentes teóricos sobre el concepto «teen series» no han permitido definir este tipo de ficción seriada como un género autónomo e independiente dentro del género programático de la ficción. No obstante, es posible destacar algunos elementos fundamentales del macrogénero ficcional ${ }^{3}$ que se pueden

${ }^{1}$ Este trabajo se ha desarrollado en el proyecto SEJ2006-10067 «Estudio de la Programación de la TV digital: innovación en contenidos, formatos y técnicas de programación en la TDT (EPTVD)» del Plan Nacional I+D+I, financiado por el Ministerio de Educación y Ciencia; y, también con el soporte del Comissionat per a Universitats i Recerca del Departamento de Innovació, Universitat i Empresa de la Generalitat de Catalunya y del Fondo Social Europeo.

2 Euromonitor es un observatorio permanente de la televisión en Europa, operativo desde 1989 y creado por un grupo de investigadores europeos (Paolo Baldi, Ian Connell, Claus Dieter Rath y Emili Prado) a petición del servicio VQPT de la RAI. El observatorio realiza informes regulares para los principales operadores televisivos de Europa y de América del Norte, así como publicaciones científicas y seminarios académicos, talleres de programación con la industria y asesoramiento a las autoridades reguladoras.

${ }^{3}$ Es posible clasificar los géneros de la ficción televisiva en muchas maneras. Por ejemplo Creeber (2001) indica tres tipos principales, cada uno de los cuales contiene más subgéneros: «drama» («single play», western, acción, policíacas, hospitalarias, ciencia ficción, drama-documental, miniserie, «teen series», etc.), «soap opera» y comedia 
atribuir también a dichos productos, como las siguientes funciones sociales: narrativa, fabuladora, barda, ritual, modeladora, familiarizadora, comunitaria, socializadora e identitaria (Casetti \& Villa, 1992; Casetti, 1992; Buonanno, 1999; Bechelloni, 1992; Fiske \& Hartley, 1978; Arnett \& otros, 1995, Browne, 1992 y Signorelli \& Lears, 1992, los tres últimos citados por Keddie \& Churchill, 1999).

El análisis descriptivo y exploratorio sobre las «teen series» producidas a partir de los años noventa, momento en el que se ocasiona una expansión significativa en la producción de las mismas, permite singularizar este tipo de ficción. En primer lugar destaca el denominador común de las «teen series», es decir el «target» adolescente al que se dirigen, y en segundo término su relación con la «teen TV» genérica, es decir el conjunto de productos televisivos y multimediáticos expresamente pensados para y dirigidos a los «teenagers». Resumiendo los hallazgos de dicho análisis, las «teen series» se pueden considerar productos de ficción seriada, generalmente de corte dramático, dirigidos principal y específicamente al público juvenil, de entre 40 y 60 minutos de duración, producidos a partir de la década de los noventa especialmente en países anglófonos y que narran las historias y las vidas de personajes adolescentes. Pueden tener un único personaje o un grupo que se constituye como protagonista, se centran en la época de la «high school», cuando los personajes tienen entre 15 y 18 años, y se caracterizan por la centralidad de tramas sobre relaciones interpersonales, especialmente de amor y amistad, como asegura también Guarinos (2009).

Respecto al concepto de adolescencia cabe destacar la dificultad de definir y delimitar un fenómeno tan complejo como culturalmente variable. En el concepto «adolescencia» tal como lo entendemos hoy en día en las sociedades occidentales han influido diferentes áreas de conocimiento, desde la educación a la biología, desde la psicología a la sociología, y más tarde la comunicación, entre algunas otras. Con el objetivo de combinar las contribuciones provenientes de los múltiples ámbitos considerados, se ha entendido la adolescencia como un proceso psicosocial, marcado por cambios físicos, psicológicos y sociales, que suele empezar con la llegada de la pubertad y culmina en la formación de un individuo adulto. La UNICEF («United Nations Children Fund») la considera una etapa fundamental de la vida y la define como «un período de transición entre la infancia y la edad adulta y, por motivos de análisis, puede segmentarse en tres etapas: adolescencia temprana (de 10 a 13 años de edad), mediana (14-16), y tardía (17-19)» (www.unicef.org/spanish/adolescence). Este estudio se ha centrado en las etapas mediana y tardía definidas por la UNICEF, indicando los individuos de edad comprendida entre 14 y 19 años con los términos «adolescentes», «jóvenes» o «teenagers» indistintamente. Se ha decidido descartar la adolescencia temprana, porque cada vez más está siendo analizada como objeto de estudio independiente, en relación a los nuevos fenómenos de consumo desarrollados por los preadolescentes, los llamados «tweens» (Ekström \& Tufte, 2007). Además, la franja de

(«sketch comedy», sitcom, animación para adultos). Euromonitor distingue trece géneros: serie, serial, «TVmovie», sitcom, miniserie, cine, cortometraje, drama (teatro), corto de animación, largo de animación, «TVmovie» de animación, serial de animación, serie de animación (Prado et al., 2009). 
edad comprendida entre los 14 y los 19 años es la que suele ser representada en las «teen series». La metodología del estudio incluye la exploración de las investigaciones de carácter internacional sobre recepción juvenil de las series juveniles de televisión, publicadas en el periodo 1981-2009, a partir de la siguiente categorización temática: a) estudios realizados en el marco del efecto cultivo; b) estudio sobre las motivaciones; c) apropiación del significado de las series y de los seriales por parte de los jóvenes; d) construcción de las identidades de los adolescentes y e) consumo y usos de los productos de ficción seriada.

\section{ADOLESCENTES, FICCIÓN TELEVISIVA Y ENTORNO MULTIMEDIÁTICO}

La aproximación a los medios de comunicación y a los adolescentes se ha analizado en numerosas ocasiones a partir de dos concepciones previas: una relacionada al pesimismo cultural y a un cierto alarmismo social, que parece remontar a las preocupaciones sobre los efectos negativos de los poetas en los jóvenes que ya Platón expresaba en la Republica; y otra relacionada con un cierto optimismo cultural, como en la tradición de los «cultural studies» o de los usos y gratificaciones (Drotner, 2000). Posiblemente la labor que se ha realizado y se viene realizando desde la «media literacy» (Von Feilitzen, 2008; Pérez Tornero, 2008; Aguaded Gómez, 2009) permita redefinir la conceptualización de la relación entre los medios y sus consumidores. Pese a ello e independientemente del punto de vista o de la perspectiva adoptada en las diferentes investigaciones, hay que destacar la abundancia de los estudios sobre los menores y los medios de comunicación, que se pueden tipificar en las siguientes áreas 4 : a) efectos positivos o negativos de los medios de comunicación, b) usos y gratificaciones, c) preferencias y motivaciones de los jóvenes, d) «agenda setting», e) teoría del cultivo, f) consumo de medios en contextos específicos de recepción, g) menores como consumidores, h) jóvenes y NTIC (Nuevas Tecnologías de la Información y Comunicación), i) los medios en la socialización de los menores, j) función educativa de los medios, y k) programas y productos para los jóvenes.

Si tradicionalmente, como afirma Buckingham (1998), ha prevalecido un cierto pánico apocalíptico, más recientemente se ha difundido una visión más positiva de la relación entre medios y menores, según la cual «children are seen to possess a powerful form of 'media literacy'» (Buckingham, 1998: 557). La rápida y enorme difusión entre los niños y los adolescentes de las NTIC ha vuelto a encender la polémica. Las nuevas generaciones de jóvenes han nacido y crecido en un entorno multimediático, donde reciben constantemente estímulos de las muchas pantallas de diferentes medidas y funciones que los envuelven: televisores de nueva generación, ordenadores, consolas o

4 Las áreas se han obtenido cruzando las líneas de investigación sobre medios y menores propuestas por Aimiller (1989), Wimmer \& Dominick (1996), Villa \& Vittadini (2005), Ekström \& Tufte (2007). 
móviles, entre otros. Estos jóvenes han sido etiquetados como «digital generation», «children in the information age», «computer nerds», «innocents on the Net», «digital divide», «addicted surfers», «generation $Y »$, «generación punto com» $\mathrm{o}$ «generación red»: ¿víctimas de los cada vez más invasivos medios de comunicación o «smart cyber-citizen of tomorrow» (Buckingham \& Sefton-Green, 1999)? Se repite una vez más la ya famosa disputa entre apocalípticos e integrados que ya Eco (1964) había intentado superar. Al margen del debate entre los nuevos apocalípticos e integrados, hay que destacar que estos jóvenes - «mediated childhoods» que viven en un «changing media environment» (Livingstone, d'Haenens \& Hasebrink, 2001) - mantienen una dieta mediática compleja, en la que entran cada vez más productos mediáticos de circulación global. Son usuarios «multitaskers», cada vez más propensos a un consumo mediático autónomo e independiente respecto al resto de la familia. Se trata del fenómeno que Bovill \& Livingstone (2001) han descrito como «bedroom culture».

El consumo mediático de los jóvenes tiene que ser analizado en relación a sus dietas recreativas, siendo el uso de los medios de comunicación sólo una de las múltiples actividades que los adolescentes desarrollan en su tiempo libre. En particular, cabe destacar que pudiendo elegir una actividad de ocio, los jóvenes prefieren socializarse, específicamente «estar» o «salir» o «hablar con los amigos», como confirman muchos estudios realizados (Funes, 2008; Gauntlett \& Hill, 1999, citados por Davis \& Dickinson, 2004; Suess et al., 1998; Garitaonandia et al., 1998; Ramírez de la Piscina et al., 2006; Casas et al., 2007; Fernández Calvià, 2005).

Por lo que concierne la dieta mediática de las nuevas generaciones, la televisión sigue teniendo un cierto peso, pero va perdiendo terreno respecto a los «new media». Efectivamente, los adolescentes son el segmento de público que menos televisión consume y que más selecciona.

Entre los contenidos televisivos favoritos de los adolescentes, la ficción ocupa un lugar privilegiado (von Feilitzen, 2004; Livingstone et al., 1998), aunque también otras tipologías de programas entran en las preferencias de los jóvenes, como la música o el «info-show» (van der Voort et al., 1998).

En el ámbito de la investigación comparativa europea «Young People, New Media» dirigida por Sonia Livingstone, se concluye que los jóvenes de países diferentes tienden a tener gustos televisivos parecidos y que la ficción suele ser el género favorito (Garitaonandia et al., 1998: 149). En particular, en la franja de edad 15-16, los chicos prefieren el deporte $(22 \%)$, las comedias $(16 \%)$, las series $(16 \%)$, la ciencia ficción (12\%) y los dibujos $(8 \%)$, mientras que las chicas prefieren las series $(33 \%)$, las «soap operas» $(28 \%)$, las comedias $(14 \%)$, la ciencia ficción (5\%) y la música $(4 \%)$.

Por lo que se refiere al contexto español, Funes (2008) proporciona las siguientes respuestas relativas a las preferencias sobre los contenidos televisivos de los jóvenes: series $(21 \%)$; deporte $(14 \%)$; películas $(12 \%)$; informativos $(6 \%)$; telenovelas $(5 \%)$; 
documentales (4\%). Además, precisa que «la audiencia de series es mayoritariamente femenina» (Funes, 2008: 121). En el contexto español otras investigaciones destacan la preferencia de los jóvenes hacia la ficción televisiva, como las de Ramírez de la Piscina et al. (2006), Medrano Samaniego, Palacios \& Barandiaran (2007), Pindado (2006), Casas et al. (2007) o Álbero Andres (2004).

\section{ESTUDIOS SOBRE CONSUMO JUVENIL DE FICCIÓN: UNA PROPUESTA DE CATEGORIZACIÓN}

La investigación específica sobre la recepción adolescente de series y seriales de ficción ha ido asumiendo cada vez más relevancia en las últimas décadas, probablemente también en consonancia con el aumento de la producción de ficción específicamente dirigida a este «target». No obstante, la mayoría de trabajos sobre recepción de ficción se ha centrado en otros grupos (como las mujeres o los estudiantes universitarios) y en particular en el género de la «soap opera».

Las primeras investigaciones empíricas sobre seriales televisivos y jóvenes se pueden situar en los Estados Unidos al principio de los años ochenta (Montero Rivero, 2006). Se trata prioritariamente, de estudios de carácter cuantitativo que se mueven alrededor de dos polos: el efecto cultivo y las motivaciones para ver «soap operas». En Europa, en cambio, destacan, en la segunda mitad de la misma década, algunos estudios cualitativos realizados en el Reino Unido. A partir de los años noventa, se aprecia la presencia de estudios realizados en varios países con metodologías mixtas, es decir con la combinación de técnicas cuantitativas y cualitativas. Sobre todo en la última década se pueden encontrar investigaciones específicas sobre el consumo de series de ficción juveniles por parte del público adolescentes, como los trabajos sobre la audiencia de «Beverly Hills 90210» de Klitgaard Povsen (1995), McKinley (1997) o Haag (1997), o sobre la recepción de la «sèrie collège» francesa «Hélène et les garçons» de Pasquier (1999). En el contexto español, destacan los estudios de recepción sobre les series españolas «Compañeros» (Huertas \& França, 2001) y «Al salir de clase» (Montero Rivero, 2005).

La categorización de los estudios que aquí se propone (Tabla 1) se basa en la identificación del aspecto principal tratado por cada trabajo, no excluyendo que cada investigación haya podido ocuparse también de otros de los factores utilizados en la clasificación.

Tabla 1. Estudios específicos sobre recepción juvenil de la ficción seriada.

\begin{tabular}{|l|l|l|l|l|l|}
\hline AUTOR & PAÍS & PERIODO & MUESTRA & $\begin{array}{l}\text { MĖTODO- } \\
\text { LOGÍA }\end{array}$ & CATEGORIA \\
\hline $\begin{array}{l}\text { Buerkel-Rothfuss \& } \\
\text { Mayes }\end{array}$ & EEUU & $<1981$ & $\begin{array}{l}\text { 290 estudiantes } \\
\text { universitarios }\end{array}$ & Cuantitativa & Efecto cultivo \\
\hline
\end{tabular}




\begin{tabular}{|c|c|c|c|c|c|}
\hline Hobson & Reino Unido & $1983 / 84$ & $\begin{array}{l}\text { Chicos de } 16 \text { años en el } \\
\text { paro }\end{array}$ & Cualitativa & Significado \\
\hline Alexander & EEUU & $<1984$ & $\begin{array}{l}230 \text { adolescentes de } 10 \text { a } \\
14 \text { años }\end{array}$ & Cuantitativa & Efecto cultivo \\
\hline Perse & EEUU & 1984 & $\begin{array}{l}458 \text { estudiantes } \\
\text { universitarios }\end{array}$ & Cuantitativa & Efecto cultivo \\
\hline Babrow & EEUU & 1984 & $\begin{array}{l}286 \text { estudiantes } \\
\text { universitarios }\end{array}$ & Cuantitativa & Motivaciones \\
\hline Rubin & EEUU & $<1985$ & 1023 estudiantes & Cuantitativa & $\begin{array}{l}\text { Consumo/Uso } \\
\mathrm{s}\end{array}$ \\
\hline Buckingham & Reino Unido & 1986 & 60 jóvenes de 7-18 años & Cualitativa & $\begin{array}{l}\text { Consumo/Uso } \\
\mathrm{s}\end{array}$ \\
\hline Gillespie & Reino Unido & $1988 / 91$ & $\begin{array}{l}333 \text { jóvenes panjabis de } \\
12-18 \text { años }\end{array}$ & Cualitativa & Identidad \\
\hline Potter & EEUU & $<1990$ & $\begin{array}{l}308 \text { estudiantes de 11-18 } \\
\text { años }\end{array}$ & Cuantitativa & Efecto cultivo \\
\hline Pasquier & Francia & $1993 / 98$ & $\begin{array}{l}\text { Más de } 700 \text { jóvenes de 8- } \\
20 \text { años }\end{array}$ & Mixta & $\begin{array}{l}\text { Consumo/Uso } \\
\mathrm{s}\end{array}$ \\
\hline Klitgaard Povsen & Dinamarca & $1993 / 96$ & $\begin{array}{l}22 \text { jóvenes de } 9-22 \text { años }+ \\
\text { más de } 300 \text { jóvenes de } 16- \\
20 \text { años }\end{array}$ & Cualitativa & $\begin{array}{l}\text { Consumo/Uso } \\
\mathrm{s}\end{array}$ \\
\hline McKinley & EEUU & 1994 & 36 chicas de 11-22 años & Cualitativa & $\begin{array}{l}\text { Consumo/Uso } \\
\mathrm{s}\end{array}$ \\
\hline Machado-Borges & Brasil & $1995 / 2000$ & $\begin{array}{l}32 \text { jóvenes de } 14-30 \text { años } \\
+183 \text { estudiantes de 11-22 } \\
\text { años }\end{array}$ & Cualitativa & $\begin{array}{l}\text { Consumo/Uso } \\
\mathrm{s}\end{array}$ \\
\hline Barker \& Andre & Reino Unido & 1996 & 77 jóvenes de $14-15$ años & Cualitativa & Identidad \\
\hline Hobson & Reino Unido & 1996 & $\begin{array}{l}\text { Chicos presidiarios de } \\
16-20 \text { años }\end{array}$ & Cualitativa & Significado \\
\hline Haag & EEUU & $<1997$ & Chicas de 12-21 años & Cualitativa & Significado \\
\hline Moran & EEUU & 1999 & $\begin{array}{l}24 \text { chicas adolescentes } \\
\text { latinas }\end{array}$ & Cualitativa & $\begin{array}{l}\text { Consumo/Uso } \\
\mathrm{s}\end{array}$ \\
\hline Peñamarin \& Vega & España & 1999 & Chicas de 15-17 años & Cualitativa & $\begin{array}{l}\text { Consumo/Uso } \\
\mathrm{s}\end{array}$ \\
\hline De Bruin & Holanda & $>1999$ & $\begin{array}{l}4 \text { grupos de } 4 / 6 \text { chicas } \\
\text { holandesas, turcas, } \\
\text { surinamesas y marroquís }\end{array}$ & Mixta & Significado \\
\hline
\end{tabular}




\begin{tabular}{|c|c|c|c|c|c|}
\hline Götz & Alemania & $2000-2001$ & 401 jovenes de $6-1 y$ anos & Cualıtatıva & Motıvaciones \\
\hline Brooker & $\begin{array}{l}\text { EEUU y } \\
\text { Reino Unido }\end{array}$ & $<2001$ & $\begin{array}{l}27 \text { chicas } \\
\text { estadounidenses de 13-21 } \\
\text { años }+40 \text { jóvenes } \\
\text { británicos de } 12-18 \text { años }\end{array}$ & Cualitativa & $\begin{array}{l}\text { Consumo/Uso } \\
\mathrm{s}\end{array}$ \\
\hline $\mathrm{SABC}^{5}$ & Sudáfrica & $>2001$ & 1200 estudiantes & Mixta & Significado \\
\hline Theunert \& Gebel & Alemania & $<2004$ & 514 jóvenes de 9-15 & Mixta & $\begin{array}{l}\text { Consumo/Uso } \\
\mathrm{s}\end{array}$ \\
\hline Quin & Australia & $<2004$ & Chicas de 12-14 años & Cualitativa & Motivaciones \\
\hline Hobson & Reino Unido & 2004 & $\begin{array}{l}3 \text { grupos de chicas de } 13, \\
14 \text { y } 17 / 18 \text { años }\end{array}$ & Cualitativa & Significado \\
\hline Montero Rivero & España & $<2006$ & $\begin{array}{l}546 \text { adolescentes de 15-16 } \\
\text { años }\end{array}$ & Cuantitativa & Significado \\
\hline \multicolumn{6}{|c|}{ Fuente: elaboración propia } \\
\hline
\end{tabular}

Más bien, los confines entre las categorías establecidas se difuminan a menudo, también en un mismo estudio, tanto por la complejidad de los trabajos analizados, como por la misma naturaleza compleja y multifacética de la relación entre adolescentes y ficción seriada. El eje central de los trabajos publicados ha permitido agrupar los estudios en cinco grandes categorías: a) efecto cultivo, b) motivaciones, c) apropiación del significado otorgado a las series y a los seriales por parte de la audiencia juvenil, d) identidad, y e) usos y consumo.

La primera categoría se refiere exclusivamente a estudios cuantitativos realizados en los Estados Unidos a principios de los años ochenta, momento en el que la teoría del cultivo vivía su auge. Estos trabajos tienen como objetivo principal comprobar, averiguar o medir el efecto cultivo generado en estudiantes universitarios a partir del visionado o el consumo de «soap operas». Por ejemplo se indagan las percepciones sobre la presencia en la sociedad de algunas categorías profesionales/no profesionales como el personal médico, la abogacía, los policías y las amas de casa, entre otros (Buerkel-Rothfuss \& Mayes, 1981; Carveth \& Alexander, 1985), y sobre eventos o relaciones sociales/sentimentales como divorcios, matrimonios, etc. (Buerkel-Rothfuss \& Mayes, 1981; Alexander, 1985). Además se producen otros estudios a partir del efecto cultivo en relación a las actitudes y a los comportamientos de los espectadores hacia las «soap operas» (Perse, 1986).

La segunda categoría, los trabajos sobre las motivaciones, comprende estudios realizados desde ambas perspectivas, la cuantitativa y la cualitativa. Estas

5 South Africa Broadcasting Corporation 
investigaciones tienen como foco central la búsqueda y el análisis de las razones y las motivaciones que mueven a los jóvenes a consumir las series de ficción o las «soap operas» (Babrow, 1987; Götz, 2004). Otros trabajos publicados centran su interés en las gratificaciones y los placeres que obtienen de su consumo (Quin, 2004). Es importante mencionar que algunos de los estudios sobre el efecto cultivo también han identificado los motivos o las gratificaciones del consumo de ficción por parte de los jóvenes.

El significado que los adolescentes otorgan a los productos de ficción seriada sintetiza la tercera categoría. Son estudios, mayoritariamente cualitativos, que indagan qué representan las series o los seriales de ficción para los jóvenes. En particular, investigan la apropiación de significados por parte de la audiencia joven (De Bruin, 2001), cómo estos construyen el significado de dichos productos (Haag, 1997), qué visiones y opiniones tienen los adolescentes sobre esos productos (Hobson, 2004), o cuáles son las interpretaciones o los valores que los jóvenes extraen de las series (Montero Rivero, 2005).

Los estudios de recepción sobre identidad y adolescencia dan nombre a la cuarta categoría. Son estudios minoritarios si se comparan con las categorías anteriores, realizados prioritariamente con técnicas cualitativas, que arrojan resultados sobre el consumo de series y seriales de ficción televisiva y los procesos de construcción de identidad de los jóvenes (Gillespie, 1995; Barker, 1997; Huertas \& França, 2001).

El último y más nutrido grupo de investigaciones reúne las investigaciones centradas en el uso y consumo. Bajo esta categoría se incluyen trabajos sobre la situación del visionado (cómo, cuándo o con quién los jóvenes miran las series), los usos posibles que los adolescentes hacen de los productos de ficción y de sus contenidos, las funciones que dichas series ejercen en el público joven, las valoraciones realizadas por los propios adolescentes en materia de ficción, y cómo los jóvenes integran el consumo de las series en la propia vida cotidiana (Lemish, 1985; Rubin, 1985; Buckingham, 1987; Pasquier, 1999; Klitgaard Povsen, 1996; McKinley, 1997; Moran, 2003; Theunert \& Gebel, 2004).

\section{CONCLUSIONES}

Las contribuciones y los hallazgos de las investigaciones publicadas en el ámbito internacional sobre la recepción juvenil de productos de ficción seriada han permitido establecer una serie de indicadores y características sobre el consumo de los adolescentes, entre los que se pueden destacar las siguientes consideraciones.

Los jóvenes suelen atribuir a, y encontrar en, los productos de ficción seriada algunas funciones fundamentales (von Feilitzen, 2004: 41): en primer lugar este «target» 
otorga a la ficción la función de entretenimiento, pero también le atribuye la función informativa y diversas funciones sociales.

El entretenimiento está relacionado con el placer o la excitación provocados por una serie específica o por un tipo específico de series (por ejemplo, las «soap operas»). Además se puede vincular al escapismo de la realidad, a una forma de combatir el aburrimiento, o más sencillamente a una manera de relajarse, o puede estar ligado a la relación de identificación entre el público joven y los personajes de las series.

La función informativa según la audiencia juvenil investigada en los estudios analizados se refiere a todo tipo de exploración de la realidad, conocimiento y aprendizaje sobre ella, sobretodo en áreas en las que los jóvenes son más inexpertos y requieren información: roles sociales, relaciones interpersonales y otros temas de índole amoroso y sexual.

Las funciones sociales se refieren no sólo a la organización temporal del menú mediático y cotidiano, sino sobretodo a la capacidad de las series de estimular y aumentar las interacciones sociales. El visionado de este tipo de ficción puede ser interpretado como una oportunidad para socializarse, al tiempo que aporta temas de conversa y opiniones en las relaciones sociales ya consolidadas de los adolescentes.

Los jóvenes son una audiencia capaz de reflexionar sobre los productos audiovisuales consumidos. Su capacidad de reflexión e interpretación críticas, realizadas según sus propios «filtros» culturales, en ocasiones les genera un distanciamiento respecto a las series. Este hecho provoca que una parte de la audiencia juvenil pueda convertirse en espectador activo y crítico de los productos de ficción seriada. Los adolescentes también pueden establecer relaciones «parasociales» con los personajes, identificarse o alejarse de ellos, descubrir como funcionan los comportamientos de esos personajes en las situaciones presentadas, reflexionar sobre sus propios comportamientos y opiniones, y comparar comportamientos reales con situaciones representadas en determinadas ficciones. Otro fenómeno destacado en los estudios es la influencia de la representación de los contenidos de las series, principalmente por lo que se refiere a personajes, temáticas, estereotipos y valores, en los procesos de construcción de las identidades, tanto individuales como colectivas. Las series y seriales dirigidos a una audiencia juvenil utilizan personajes de ese mismo «target», precisamente por la satisfacción de ver en la pantalla personas de edades similares a las de su público (Harwood, 1997).

La compleja relación que existe entre los adolescentes y los productos de ficción seriada no sólo está presente en las aproximaciones científicas, sino que también es y sigue siendo preocupación empresarial y desconocimiento de los operadores. En un mundo globalizado e interconectado, el consumo de productos audiovisuales requiere el desarrollo de capacidades y habilidades por parte de las audiencias. La recepción de la comunicación mediática se puede entender como una función experta, que pone en juego 
las «enciclopedias» de referencia de los destinatarios, sus conocimientos, capacidades y expectativas. Se trata entonces de una interpretación activa y rutinaria, contextualizada socialmente, de una apropiación subjetiva a través de la cual los receptores integran los mensajes mediáticos en sus procesos de entendimiento (Salzano, 2003). Además, tener un buen conocimiento de qué prefieren los jóvenes, de cuáles son sus expectativas y preferencias relativas a la ficción televisiva, de cómo incorporan el consumo de ficción en su dieta multimediática y en su vida cotidiana, puede ayudar a diseñar productos específicos para ellos, que sean capaces de atraerlos y entretenerlos además de ser educativos. La serie juvenil «Yizo Yizo» («Así son las cosas»), producida y emitida en Sudáfrica entre 1999 y 2004, es un buen ejemplo de ello (Smith, 2004; Barnett, 2004), pero sólo uno de los pocos.

Precisamente en un momento clave del desarrollo de los medios a partir de la digitalización, la consecuente proliferación de canales y la clara apuesta por los canales temáticos de ficción, se revaloriza el conocimiento sobre el consumo adolescente de la ficción seriada. Y por ello su estudio desde el ámbito académico es todavía más urgente.

\section{BIBLIOGRAFÍA}

- AGUADED GÓMEZ, Jose Ignacio (2009). El Parlamento Europeo apuesta por la alfabetización mediática. En: Comunicar, 32, págs. 7-8.

- AIMILLER, Kurt (1989). Television and young people: a bibliography of international literature 1969-1989. München [etc.]. K.G. Saur.

- ÁLBERO ANDRES, Magdalena (2004). Violència, sexe I televisió: la mirada adolescent. Treball d'investigació: La incidència dels continguts televisius de violència I sexe en el comportament dels adolescents. Conveni de collaboració entre el Consell Audiovisual de Catalunya i la Universitat Autònoma de Barcelona.

- ALEXANDER, Alison (1985). Adolescents' soap opera viewing and relational perceptions. En: Journal of Broadcasting and Electronic Media, 29 (3), págs. 295-308.

- BABROW, Austin S. (1987). Students' motives for watching soap opera. En: Journal of Broadcasting and Electronic Media, 31 (3), págs. 309-321.

- BARKER, Chris (1997). Television and the reflexive project of the self: soap, teenage talk and hybrid identity. En: The British Journal of Sociology, 48 (4), págs. 611-628.

- BARNETT, Clive (2004). Yizo Yizo: citizenship, commodification and popular Culture in South Africa. En: Media Culture \& Society, 26, págs. 251-271. 
- BECHELLONI, Giovanni (1992). La televisione racconta. En CASETTI, Francesco; VILLA, Federica (ed): La storia comune: funzioni, forme e generi della fiction televisiva. Torino. Nuova Eri.

- BOVILL, Moira; LIVINGSTONE, Sonia (2001). Bedroom culture and the privatization of media use. En LIVINGSTONE, Sonia; BOVILL, Moira (ed.)(2001): Children and their changing media environment. A European comparative study. Mahwah, New Jersey, London. Lawrence Erlbaum Associates, Publishers.

- BUCKINGHAM, David (1987). Public secrets: Eastenders and its audience. London. BFI.

- BUCKINGHAM, David (1998). Review essay: Children of the electronic age? Digital media and new generation rhetoric. En: European Journal of Communication, 14 (4), págs. 557-565.

- BUCKINGHAM, David; SEFTON-GREEN, Julian (1999). Children, Young People and Digital Technology. En: Convergence 5; 4.

- BUERKEL-ROTHFUSS, Nancy; MAYES, Sandra (1981). Daytime serial drama. Soap opera viewing: the cultivation effect. En: Journal of Communication, 31, págs.108-115.

- BUONANNO, Milly (1999). El drama televisivo. Contenidos y temas sociales. Barcelona. Gedisa.

- CARVETH, Rodney; ALEXANDER, Alison (1985). Soap opera viewing motivations and the cultivation process. En: Journal of Broadcasting and Electronic Media, 29 (3), págs. 259-273.

- CASAS, Ferran et al. (2007). Preferències $i$ expectatives dels adolescents relatives a la televisió a Catalunya. CAC i Universitat de Girona.

- CASETTI, Francesco (1992). Le funzioni della fiction televisiva. En CASETTI, Francesco; VILLA, Federica (ed): La storia comune: funzioni, forme e generi della fiction televisiva. Torino. Nuova Eri.

- CASETTI, Francesco; VILLA, Federica (ed) (1992). La storia comune: funzioni, forme e generi della fiction televisiva. Torino. Nuova Eri.

- CREEBER, Glen (ed.) (2001). The television genre book. London. BFI. 
- DAVIS, Glyn; DICKINSON, Kay (ed.) (2004). Teen TV: genre, consumption, identity. London. BFI.

- DE BRUIN, Joost (2001). Dutch television soap opera, ethnicity and girls' interpretation. En: Gazette, 63 (1), págs. 41-56.

- DROTNER, Kristen (2000). Difference and diversity: trends in young Danes' media use. En: Media, Culture \& Society, 22 (2), págs. 149-166.

- ECO, U. (1964). Apocalittici e integrati. Comunicazioni di massa e teorie della cultura di massa. Milano. Bompiani.

- EKSTRÖM, Karen M.; TUFTE, Birgitte (ed.) (2007). Children, media and consumption. On the front edge. Göteborg: International Clearinghouse on Children, Youth and Media, Nordicom, Göteborg University.

- FERNÁNDEZ CALVIÀ, Josep (2005). La Pantalla Amiga? Nens i Adolescents davant el nou entorn audiovisual. Tarragona. Arola Editors.

- FISKE, John; HARTLEY, John (1978). Reading Television. London. Methuen \& Co.

- FUNES, María Jesús (2008). Cultura, Política y Sociedad. En INJUVE (2008). Informe de la Juventud en España. Gobierno de España, Ministerio de Igualdad.

- GARITAONANDIA, Carmelo; JURASTI, Patxi; PASTOR, Fatima; OLEAGA, José A. (1998). Las relaciones de los niños y de los jóvenes con las viejas y nuevas tecnologías de la información. En: ZER no 4, págs. 131-161.

- GILLESPIE, Marie (1995). Television, ethnicity and cultural change. London. Routledge.

- GÖTZ, Maya (2004). "Soaps want to explain reality". Daily soaps and Big Brother in the everyday life of German children and adolescents. En: VON FEILITZEN, Cecilia (ed.) (2004). Young People, Soap Operas and Reality TV. The International Clearinghouse on Children, Youth and Media. Nordicom, Götenborg University.

- GUARINOS, Virginia (2009). Fenómenos televisivos «teenagers»: prototipias adolescentes en series vistas en España. En: Comunicar, 33, págs. 203-211.

- HAAG, Darcy (1997). Using Beverly Hills, 90210 to explore developmental issues in female adolescents. En: Youth Society, 1, págs. 24-53. 
- HOBSON, Dorothy (2004). Everyday people, everyday life. British teenagers, soap opera and reality TV. En VON FEILITZEN, Cecilia (ed.)(2004). Young People, Soap Operas and Reality TV. The International Clearinghouse on Children, Youth and Media. Nordicom, Götenborg University.

- HARWOOD, Jake (1997). Viewing age: lifespan identity and television viewing choices. En: Journal of Broadcasting and Electronic Media, 41, págs. 203-213.

- HUERTAS, Amparo; FRANÇA, Maria E. (2001). El espectador adolescente. Una aproximación a cómo contribuye la televisión en la construcción del yo. ZER, 11 (http://www.ehu.es/zer/) (12-01-09).

- KEDDIE, Amanda; CHURCHILL, Rick (1999). Young adolescents' gender-related perceptions of TV characterizations. En: Australian Journal of Communication, 26 (1), págs. 87-106.

- KLITGAARD POVSEN, Karen (1996). Global teen soaps go local: Beverly Hills 90210 in Denmark. En: Young, Nordic Journal of Youth research, 4 (4), págs. 3-20.

- LEMISH, Dafna (1985). Soap opera viewing in college: a naturalistic inquiry. En: Journal of Broadcasting and Electronic Media, 29 (3), págs. 275-293.

- LEMISH Dafna; DROTNER, Kirsten; LIEBES, Tamar; MAIGRET, Eric; STALD, Gitte (1998). Global culture in practice. A look at children and adolescents in Denmark, France and Israel. En: European Journal of Communication, 13 (4), págs. 539-556.

- LIVINGSTONE, S.; D'HAENENS, L. \& HASEBRINK, U. (2001). Childhood in Europe: Contexts for Comparition. En LIVINGSTONE, Sonia; BOVILL, Moira (ed.)(2001): Children and their changing media environment. A European comparative study. Mahwah, New Jersey, London. Lawrence Erlbaum Associates, Publishers.

- MCKINLEY, E. Graham (1997). Beverly Hills 90210: Television, gender and identity. Philadelphia. University of Pennsylvania Press.

- MEDRANO SAMANIEGO, Concepción; PALACIOS, Santiago \& BARANDIARAN, Ana A. (2007). Los hábitos y preferencias televisivas en jóvenes y adolescentes: Un estudio realizado en el País Vasco. En: Revista Latina de Comunicación Social, 62 (http://www.ull.es/publicaciones/latina/200702Medrano_S_yotros.pdf) (12-0109). 
- MONTERO RIVERO, Yolanda (2005). Estudio empírico sobre el serial juvenil «Al salir de clase»: sobre la transmisión de valores a los adolescentes. En: Comunicar, 25 (2) (http:/ / www.revistacomunicar.com/verpdf.php?numero=25\&articulo=25-2005070) (12-01-09).

- MONTERO RIVERO, Yolanda (2006). Televisión, valores y adolescencia. Barcelona. Gedisa.

- MORAN, Kristin C. (2003). A Reception Analysis: Latina Teenagers Talk About Telenovelas. En: Global Media Journal, American Edition, 2(2) (http:/ /lass.calumet.purdue.edu/cca/gmj/sp03/gmj-sp03-moran.htm) (12-01-09).

- PASQUIER, Dominique (1999). La culture des sentiments: l'expérience télévisuelle des adolescentes. Paris. Éditions de la Maison des sciences de l'homme.

- PÉREZ TORNERO, José Manuel (2008). Media Literacy. New Conceptualisation, New Approach. En VVAA (eds.) (2008). Empowerment Through Media Education. An Intercultural Dialogue. Götenborg. Nordicom.

- PERSE, Elizabeth M. (1986). Soap opera viewing patterns of college students and cultivation. En: Journal of Broadcasting and Electronic Media, 30 (2), págs. 175-193.

- PINDADO, Julián (2006). Los medios de comunicación y la construcción de la identidad adolescente. En: Zer, 21, págs. 11-22.

- PRADO, Emili; FRANQUET, Rosa; RIBES, Xavier; SOTO, Maite; FERNÁNDEZ QUIJADA, David (2006). Televisió Interactiva. Simbiosi tecnologica i sistemes d'interacció amb la televisió. Barcelona. Consell Audiovisual de Catalunya.

- PRADO, Emili; DELGADO, Matilde; GARCÍA-MUÑOZ, Núria; LARREGOLA, Gemma (2009). La televisió. En: COROMINAS, M. \& MORAGAS, M. de (eds). Informe de la Comunicació a Catalunya 2007-2008. Bellaterra. Institut de la Comunicació, Universitat Autònoma de Barcelona.

- QUIN, Robyn (2004). From Beverly Hills to Big Brother. How Australian teenage girls respond. En: VON FEILITZEN, Cecilia (ed.) (2004). Young People, Soap Operas and Reality TV. The International Clearinghouse on Children, Youth and Media. Nordicom, Götenborg University. 
- RAMÍREZ DE LA PISCINA, Txema; ZARANDONA, Esther; BASTERRETXEA, Jose Inazio; IDOIAGA, Petos (2006). Estudio sobre la alfabetización audiovisual de los adolescentes vascos: Recopilatorio de actitudes críticas y acríticas. En: Zer, 21, págs. 177202.

- RUBIN, Alan M. (1985). Uses of daytime television soap operas by college students. En: Journal of Broadcasting and Electronic Media, 29 (3), págs. 241-258.

- SALZANO, Diana (2003). Lo sguardo disancorato. Società globale e comunicazione. Napoli. Editoriale Scientifica.

- SMITH, René (2004). Yizo Yizo: This is it? A critical analysis of a reality-based drama series. En: VON FEILITZEN, Cecilia (ed.) (2004). Young People, Soap Operas and Reality TV. The International Clearinghouse on Children, Youth and Media. Nordicom, Götenborg University.

- SUESS, Daniel; SUONINEN, Annikka; GARITAONANDIA, Carmelo; JUARISTI, Patxi; KOIKKALAINEN, Riitta; OLEAGA, José A. (1998). Media use and the relationship of children and teenagers with their peer groups. A study of Finnish, Spanish and Swiss cases. En: European Journal of Communication, 13 (4), págs. 521-538.

- THEUNERT, Helga \& GEBEL, Christa (2004). Reception on TV series among children and teenagers in Germany. En: VON FEILITZEN, Cecilia (ed.) (2004). Young People, Soap Operas and Reality TV. The International Clearinghouse on Children, Youth and Media. Nordicom, Götenborg University.

- VON FEILITZEN, Cecilia (2008). Children and Media Literacy: critique, practice, democracy. En: Doxa, 6, págs. 317-332.

- VAN DER VOORT, Tom H.A.; BEENTJES, Johannes W.J.; BOVILL, Moira; GASKELL, George; KOOLSTRA, Cees M.; LIVINGSTONE, Sonia; MARSEILLE, Nies (1998). Young people's ownership and uses of new and old forms of media in Britain and the Netherlands. En: European Journal of Communication, 13(4), págs. 457-477.

- VILLA, Marina; VITTADINI, Nicoletta (2005). Media e adolescenti: un percorso tra ricerca e regolamentazione. En: IKON, Forme e processi del comunicare, 50-51, págs. $11-32$. 
- WIMMER, Roger D. \& DOMINICK, Joseph R. (1996). La investigación científica de los medios de comunicación: una introducción a sus métodos. Barcelona. Bosch. 